\title{
Evaluation of some integrals of sums involving the Möbius function
}

\author{
TADEJ KOTNIK* \\ Faculty of Electrical Engineering, University of Ljubljana, \\ Tržaška 25, SI-1000 Ljubljana, Slovenia
}

(Received 26 October 2006; revised version received 04 December 2006; accepted 28 December 2006)

\begin{abstract}
Integrals of sums involving the Möbius function appear in a variety of problems. In this paper, a divergent integral related to several important properties of the Riemann zeta function is evaluated computationally. The order of magnitude of this integral appears to be compatible with the Riemann hypothesis, and furthermore the value of the multiplicative constant involved seems to be the smallest possible. In addition, eleven convergent integrals representing Tauberian constants that characterize the relations between certain summation methods are evaluated computationally to five or more digits of precision.
\end{abstract}

Keywords: Möbius function; Riemann hypothesis; Tauberian constants; Weak Mertens hypothesis

AMS Subject Classifications: 11M06; 11Y60; 65D30

\section{Introduction}

The Möbius function $\mu(n)$ is defined as $\mu(1)=1, \mu(n)=(-1)^{k}$ if $n$ is the product of $k$ different primes, and $\mu(n)=0$ if $n$ contains a prime factor to a power higher than the first. It was introduced by August Möbius in 1832 for the purpose of inverting sums of arithmetic functions [1], and various 'Möbius inversion formulae' found important applications, among others that of Bernhard Riemann in 1859 in his derivation of an exact formula for the primecounting function [2]. Sums involving $\mu(n)$ such as

$$
Q(x):=\sum_{1 \leq n \leq x}|\mu(n)|, \quad M(x):=\sum_{1 \leq n \leq x} \mu(n), \quad \text { and } \quad g(x):=\sum_{1 \leq n \leq x} \frac{\mu(n)}{n}
$$

as well as certain integrals involving these functions, are closely related to properties of the square-free numbers, the primes, and the Riemann zeta function $\zeta(s)$. It is easily seen that

\footnotetext{
*Email: tadej.kotnik@fe.uni-lj.si
} 
$Q(x)$ counts the square-free numbers, and it is known that

$$
Q(x)=\frac{6}{\pi^{2}} x+E_{Q}(x)
$$

where $\left|E_{Q}(x)\right|<0.1333 \sqrt{x}$ for $x>1164$ [3], and $\left|E_{Q}(x)\right|<0.02767 \sqrt{x}$ for $x>$ 438653 [4]. The properties of $M(x)$, which keeps track of the balance between the squarefree numbers with an odd and those with an even number of prime factors, are understood much less precisely, as it is only known that $M(x)=\Omega\left(x^{1 / 2}\right)$ [5, Theorem 14.26B] and $M(x)=O\left(x / \log ^{236 / 75} x\right)$ [4]. The Riemann hypothesis (i.e. the conjecture that all the nonreal zeros of $\zeta(s)$ lie on the line $\Re(s)=1 / 2$ ) corresponds to a much stronger $O$-bound, namely $M(x)=O\left(x^{1 / 2+\varepsilon}\right)[5$, Theorem 14.25C]. A related conjecture known as 'the weak Mertens hypothesis'

$$
\int_{1}^{X} \frac{M^{2}(x)}{x^{2}} d x=O(\log X)
$$

would imply that the Riemann hypothesis is true, all the zeros of $\zeta(s)$ are simple, and $\zeta^{\prime}(\rho)^{-1}=$ $o(\rho)$ for every non-real zero $\rho$ of $\zeta(s)$. Recently, $\mathrm{Ng}$ showed [6] that (1) itself would follow from the Riemann hypothesis and $\sum_{0<\gamma<T}\left|\zeta^{\prime}(\rho)\right|^{-2}=O(T)$, where $\gamma$ are the imaginary parts of the zeros of $\zeta(s)$ on the line $\Re(s)=1 / 2$. Finally, from (1) it would also follow that $\sum\left|\rho \zeta^{\prime}(\rho)\right|^{-2}$ converges; more precisely, if (1) holds with an asymptotic order constant $A$, then the sum of this series is at most $A$ [5, Theorem 14.29B].

The computational evaluation of the integral in (1) is presented in section 3 , and seems to suggest that $\log X$ is indeed its order of magnitude, and that the sum of the series $\sum\left|\rho \zeta^{\prime}(\rho)\right|^{-2}$ could even be its order constant.

The functions $Q(x), M(x)$, and $g(x)$ also appear in certain convergent integrals representing Tauberian constants of the relations between certain summation methods. These integrals, first derived by Jukes $[7,8]$, will be denoted here as follows

$$
\begin{gathered}
I_{1}:=\int_{1}^{\infty}\left|\frac{Q(x)}{x^{2}}-\frac{6}{\pi^{2} x}\right| d x, \quad I_{2}:=\int_{1}^{\infty}\left|\frac{Q(x)}{x^{2}}-\frac{6[x]}{\pi^{2} x^{2}}\right| d x, \quad I_{3}:=\int_{1}^{\infty} \frac{2|M(x)|}{x^{3}} d x, \\
I_{4}:=\int_{1}^{\infty} \frac{2|M(x)-1|}{x^{3}} d x, \quad I_{5}:=\int_{1}^{\infty} \frac{|M(x)|}{x^{2}} d x, \quad I_{6}:=\int_{1}^{\infty} \frac{|g(x)|}{x} d x, \\
I_{7}:=\int_{1}^{\infty} \frac{|1-x g(x)|}{x^{2}} d x, \quad I_{8}:=\int_{1}^{\infty} \frac{|M(x)-x g(x)|}{x^{2}} d x, \\
I_{9}:=\int_{1}^{\infty} \frac{|M(x)+1-x g(x)|}{x^{2}} d x, \quad I_{10}:=\int_{1}^{\infty} \frac{|M(\sqrt{x})-x g(x)|}{x^{2}} d x, \\
I_{11}:=\int_{1}^{\infty} \frac{|M(x)+M(\sqrt{x})-x g(x)|}{x^{2}} d x .
\end{gathered}
$$

In a computational estimation, the integration is necessarily truncated from $\int_{1}^{\infty}$ to $\int_{1}^{N}$. Gretton and Jukes computed such truncations of the above integrals for $N$ up to $7.5 \times 10^{5}[9,10]$, but they only determined explicit error bounds for their estimates of $I_{1}$ and $I_{2}$. In section 4 their computations are extended significantly (to $N$ ranging from $2.2 \times 10^{7}$ to $10^{13}$, depending on the rate of convergence of the integral under consideration) and explicit error bounds are provided for all eleven numerical estimates. For five of these estimates the error bounds are unconditional, and the remaining six are based on a both theoretically and numerically plausible conjecture concerning the orders of magnitude of $M(x)$ and $g(x)$. 


\section{Methods of computation and processing}

The computations were performed with a program written and compiled in Delphi ${ }^{\mathrm{TM}} 6.0$ (Borland, Scotts Valley, CA, USA), and run on a PC with a $2.4 \mathrm{GHz}$ Intel ${ }^{\circledR}$ Pentium ${ }^{\circledR} 4$ processor and $512 \mathrm{MB}$ of RAM. The values of $\mu(n)$ were computed using the sieving algorithm described in $[11,12]$. The values of $Q(n), M(n)$, and $g(n)$ were computed according to their definitions, and the integrals involving $Q(x), M(x)$, and $g(x)$ were evaluated in steps covering intervals between consecutive integers, where the integrals can be expressed by elementary functions. As an illustration, for $I_{11}$, the increment in the interval $n \leq x<n+1$ was computed as

$$
\begin{aligned}
& \int_{n}^{n+1} \frac{|M(x)+M(\sqrt{x})-x g(x)|}{x^{2}} d x=\int_{n}^{n+1}\left|\frac{M(n)+M(\sqrt{n})}{x^{2}}-\frac{g(n)}{x}\right| d x \\
& =\left\{\begin{array}{l|l}
\mid \begin{array}{c}
\frac{(M(n)+M(\sqrt{n}))(2 n+1)}{n(n+1)} \\
-g(n)\left(2+\log \frac{(M(n)+M(\sqrt{n}))^{2}}{n(n+1) g^{2}(n)}\right)
\end{array} & \left(n<\frac{M(n)+M(\sqrt{n})}{g(n)}<n+1\right) \\
\left|\frac{M(n)+M(\sqrt{n})}{n(n+1)}-g(n) \log \frac{n+1}{n}\right| & \text { (otherwise) }
\end{array}\right.
\end{aligned}
$$

and the other integrals were treated analogously.

The values of $n$ were stored as 64-bit integers (type Int64), the values of $\mu, M$, and $Q$ as 32-bit integers (type Integer), and all non-integer variables as 80-bit reals (type Extended, 19 significant digits). To minimize the loss of significant digits at large $n$, where $\int_{1}^{n}$ is much larger than $\int_{n}^{n+1}$, for each evaluated integral one variable was used for the basic value $\int_{1}^{n_{1}}$, and another for the increment $\int_{n_{1}}^{n_{2}}$. When the increment exceeded the basic value, their sum became the basic value and the increment was set to zero, and so repeatedly through the computation. The sum of the two integrals was also determined when storing the computed values.

The values of the integral (1) were stored at all $n \leq 10^{4}$ and at all $10^{4}<n<10^{13}$ satisfying $n=\left\lfloor 10^{k / 2400}\right\rfloor$ with $k \in \mathbb{N}$. This provided a sufficiently dense set of sampled values for further analysis and plotting of the integral on a logarithmic abscissa.

The truncations of $I_{1}, I_{2}, \ldots, I_{11}$ were stored at all $n \leq 10^{4}$ and at larger $n=m \cdot 10^{k}$ with $k, m \in \mathbb{N}$ and $1 \leq m \leq 99$. The computation of each integral was halted when the desired precision of the estimate was reached; this amounted to seven digits after the decimal point for $I_{1}$ and $I_{2}$, to 10 such digits for $I_{3}$ and $I_{4}$ (all for unconditional error bounds), and to five digits after the decimal point for the other seven integrals (for conditional error bounds, see section 4 for details).

\section{The weak Mertens conjecture}

Figure 1 shows the plot of the integral (1) on a logarithmic abscissa spanning the range $1 \leq X \leq 10^{13}$, which seems to suggest that $\log X$ could well be the actual order of magnitude of this integral. For $10^{5} \leq X \leq 10^{13}$, the best correlation between the sampled data and a function $\alpha+\beta \log X$ is obtained with $\alpha=1.262 \ldots, \beta=0.02903 \ldots$ In figure 1 this function would obscure the curve of the integral (1), so its translate $1.2+0.02903 \log X$ is shown instead (dashed). 


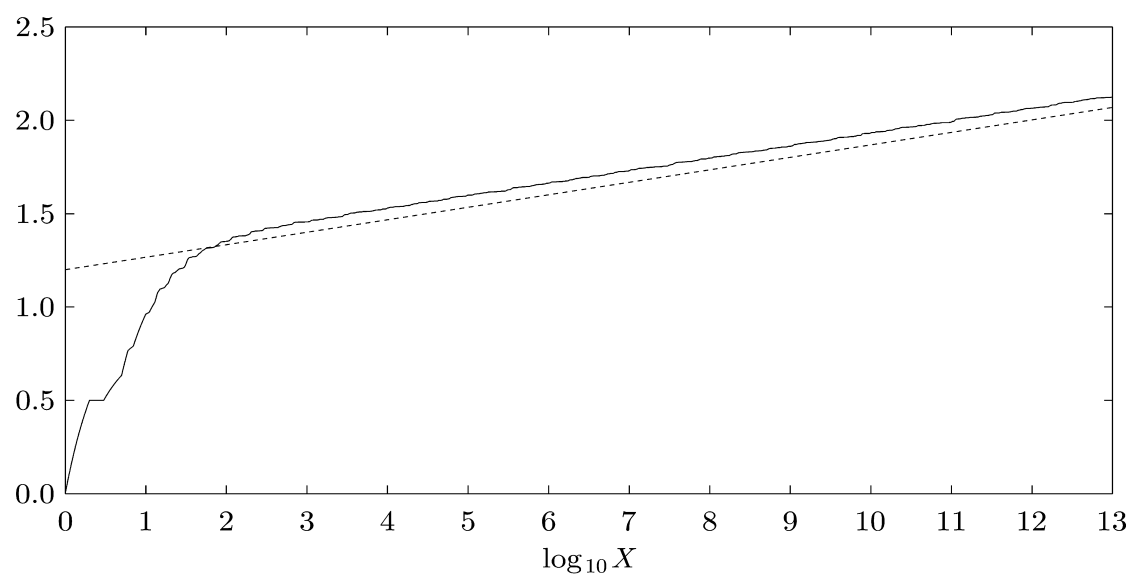

Figure 1. The integral $\int_{1}^{X}\left(M^{2}(x) / x^{2}\right) d x$ (solid) and the function $1.2+0.02903 \log X$ (dashed).

Numerical data suggest that the series $\sum\left|\rho \zeta^{\prime}(\rho)\right|^{-2}$ converges to the value $0.02903 \ldots$ (see table 5 in [13]). As mentioned in section 1, the sum of this series is also the smallest possible value of

$$
\lim _{X \rightarrow \infty} \frac{\int_{1}^{X}\left(M^{2}(x) / x^{2}\right) d x}{\log X}
$$

and from the data shown in figure 1 it seems that $0.02903 \ldots$ could be the actual value of this limit.

\section{The Tauberian constants}

Explicit upper bounds of the errors caused by truncating the integrals $I_{1}, I_{2}, \ldots, I_{11}$ from $\int_{1}^{\infty}$ to $\int_{1}^{N}$ were obtained using a number of inequalities. In 1995 El Marraki [4] proved that:

$$
\begin{gathered}
\left|Q(x)-\frac{6 x}{\pi^{2}}\right|<0.02767 \sqrt{x} \quad(x>438653) \\
|M(x)|<\frac{x}{4345} \quad(x>2160535)
\end{gathered}
$$

and from Theorem 3 of the same paper it also follows that

$$
|M(x)|<\frac{0.58782 x}{\log ^{11 / 9} x} \quad(x>685) .
$$

For brevity, we now denote $I_{k}=\int_{1}^{N}+E_{k}(N)$. Using (2) we get

$$
E_{1}(N)=\int_{N}^{\infty}\left|\frac{Q(x)}{x^{2}}-\frac{6}{\pi^{2} x}\right| d x<\int_{N}^{\infty} \frac{0.02767}{x^{3 / 2}} d x=\frac{0.05534}{\sqrt{N}} \quad(N>438653)
$$

and a similar result for $E_{2}(N)$. In the same manner (3) yields

$$
E_{3}(N)=2 \int_{N}^{\infty} \frac{|M(x)|}{x^{3}} d x<\int_{N}^{\infty} \frac{d x}{2172.5 x^{2}}=\frac{1}{2172.5 N} \quad(N>2160535)
$$


and a similar result for $E_{4}(N)$. Even for small $N$, these error bounds are considerably smaller than the truncated integrals they characterize, and they also decrease rapidly as $N$ increases.

In the other integrals involving $M(x)$, inequality (3) is too weak for a finite error bound. Inequality (4), being asymptotically slightly stronger, is just sufficient for this in the case of $I_{5}$, yielding

$$
E_{5}(N)=\int_{N}^{\infty} \frac{|M(x)|}{x^{2}} d x<\int_{N}^{\infty} \frac{0.58782}{x \log ^{11 / 9} x} d x=\frac{2.64519}{\log ^{2 / 9} N} \quad(x>685) .
$$

The obtained upper bound of $E_{5}(N)$ decreases so slowly that for any computationally feasible value of $N$ it is larger than the truncated integral, but (4) nonetheless allows us to demonstrate that $I_{5}$ is convergent, and even to obtain an upper bound of its value.

The remaining six integrals involve the function $g(x)$, for which no explicit inequality seems to have been published in the literature. Writing

$$
\begin{aligned}
g(x) & =\sum_{k=1}^{[x]} \frac{\mu(k)}{k}=\sum_{k=1}^{\infty} \frac{\mu(k)}{k}-\sum_{k=[x]+1}^{\infty} \frac{\mu(k)}{k}=0-\sum_{k=[x]+1}^{\infty} \frac{\mu(k)}{k} \\
& =\sum_{k=[x]+1}^{\infty} \frac{M(k-1)-M(k)}{k}=\frac{M([x])}{[x]+1}+\sum_{k=[x]+1}^{\infty} M(k)\left(\frac{1}{k+1}-\frac{1}{k}\right) \\
& =\frac{M(x)}{[x]+1}-\int_{[x]+1}^{\infty} \frac{M(u)}{u^{2}} d u
\end{aligned}
$$

we see that

$$
\begin{aligned}
|g(x)| & \leq \frac{|M(x)|}{x}+\left|\int_{[x]+1}^{\infty} \frac{M(u)}{u^{2}} d u\right|<\frac{|M(x)|}{x}+\int_{[x]+1}^{\infty} \frac{|M(u)|}{u^{2}} d u \\
& \leq \frac{|M(x)|}{x}+\int_{x}^{\infty} \frac{|M(u)|}{u^{2}} d u
\end{aligned}
$$

so that by (4) we get

$$
|g(x)|<\frac{0.58782}{\log ^{11 / 9} x}+\frac{2.64519}{\log ^{2 / 9} x} \quad(x>685)
$$

but due to the second term on the right-hand side this inequality is slightly too weak to provide a finite upper bound on integrals of the type $\int_{N}^{\infty}(|g(x)| / x) d x$.

It was mentioned in section 1 that the Riemann hypothesis corresponds to $M(x)=$ $O\left(x^{1 / 2+\varepsilon}\right)$. Numerical data suggest that this is considerably closer to the actual situation than the much weaker unconditional inequalities (3) and (4). From the plots of the functions $M(x) / \sqrt{x}$ (figure 2, solid), $g(x) \sqrt{x}$ (figure 3, solid), and $\pm(1 / 4) \log \log x$ (figures 2 and 3 , dashed) in the range $1 \leq x \leq 10^{14}$ it appears that

$$
|M(x)|<\frac{\sqrt{x} \log \log x}{4} \quad(x>295)
$$

and

$$
|g(x)|<\frac{\log \log x}{4 \sqrt{x}} \quad(x>222)
$$




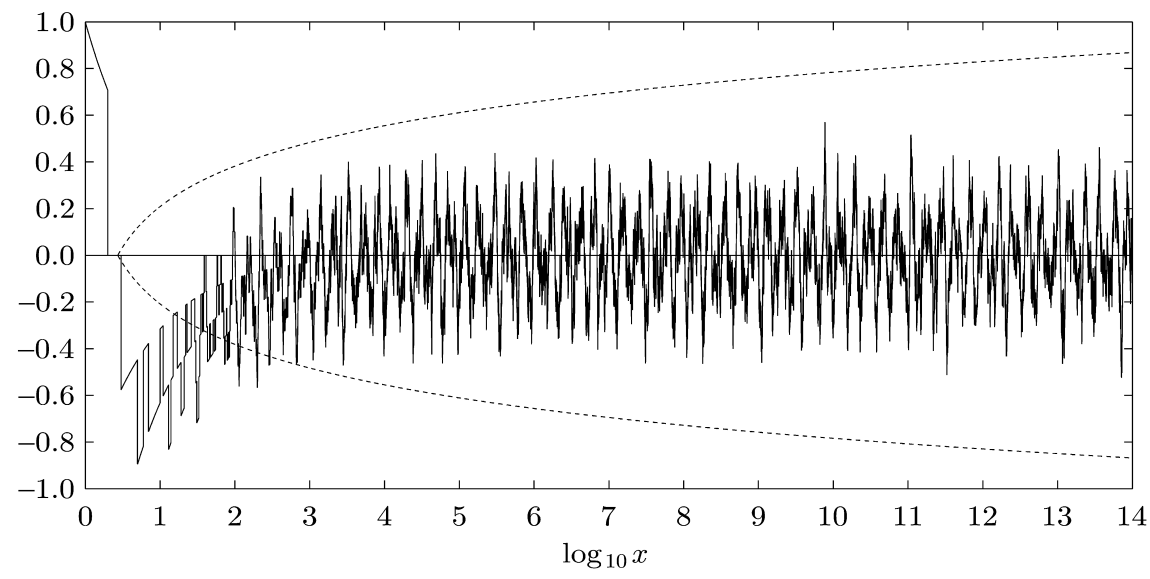

Figure 2. The functions $M(x) / \sqrt{x}$ (solid) and $\pm 0.25 \log \log x$ (dashed).

where the $O$-form of (7) also follows from (5) and (6). The inequalities (6) and (7) are asymptotically more conservative than typical conjectural estimates that have been proposed during the last decades, which are of the form $O\left(x^{1 / 2}(\log \log x)^{1 / 2}\right)[14,15]$ or even $O\left(x^{1 / 2}(\log \log \log x)^{K}\right)$ for some $K>0[6,13]$.

Under the conditions (6) and (7), upper bounds of the truncation errors are easily obtained for $I_{5}, I_{6}, \ldots, I_{11}$. Denoting these conditional error bounds by $\widetilde{E}_{k}$, we get

$$
\widetilde{E}_{5}(N)=\int_{N}^{\infty} \frac{|M(x)|}{x^{2}} d x<\frac{1}{4} \int_{N}^{\infty} \frac{\log \log x}{x^{3 / 2}} d x=\frac{\log \log N}{2 \sqrt{N}}-\frac{\operatorname{li}(1 / \sqrt{N})}{2} \quad(N>295)
$$

which decreases much more rapidly than the unconditional error bound $E_{5}(N)$, and similar expressions are obtained for the other integrals.

Table 1 summarizes the estimated values of the eleven Tauberian constants and their corresponding error bounds (unconditional where available, and conditional otherwise). The digits in larger typeface are established with certainty-unconditionally for the first four integrals (underlined) and conditionally for the rest - while the digits in smaller typeface that follow are provided for possible extension of the computations. The estimates obtained by Gretton and

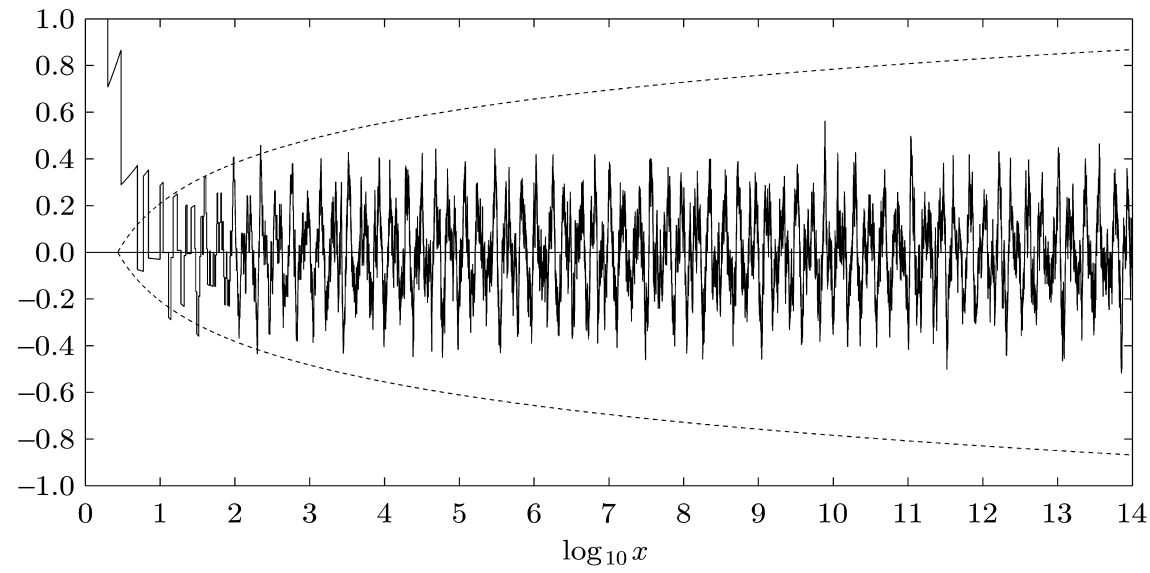

Figure 3. The functions $g(x) \sqrt{x}$ (solid) and $\pm 0.25 \log \log x$ (dashed). 
Table 1.

\begin{tabular}{|c|c|c|c|c|c|}
\hline \multirow[b]{2}{*}{$k$} & \multicolumn{2}{|c|}{ Estimate of $I_{k}$} & \multirow[b]{2}{*}{$N$} & \multicolumn{2}{|c|}{ Upper error bound } \\
\hline & {$[9,10]$} & This paper & & $E_{k}(N)$ & $\widetilde{E}_{k}(N)$ \\
\hline 1 & $0.46 \ldots$ & $\underline{0.4616041_{09} \ldots}$ & $7.3 \mathrm{e} 11$ & $6.48 \mathrm{e}-8$ & \\
\hline 2 & $0.69 \ldots$ & $\underline{\underline{0.6945017}}_{07} \ldots$ & $7.3 \mathrm{e} 11$ & $6.48 \mathrm{e}-8$ & \\
\hline 3 & $0.892 \ldots$ & $\underline{0.8921506905} 42 \ldots$ & $2.2 \mathrm{e} 7$ & $2.10 \mathrm{e}-11$ & $9.26 \mathrm{e}-12$ \\
\hline 4 & $0.392 \ldots$ & $\underline{0.3921032696}_{80} \ldots$ & $4.8 \mathrm{e} 7$ & $9.60 \mathrm{e}-12$ & $2.92 \mathrm{e}-12$ \\
\hline 5 & $1.01 \ldots$ & $1.01426_{653} \ldots$ & $7.3 \mathrm{e} 11$ & 1.26839 & $1.98 \mathrm{e}-6$ \\
\hline 6 & $1.09 \ldots$ & $1.09667_{052} \ldots$ & $7.3 \mathrm{e} 11$ & & $1.98 \mathrm{e}-6$ \\
\hline 7 & $0.483 \ldots$ & $0.483439_{40} \ldots$ & $9.0 \mathrm{e} 12$ & & $5.77 \mathrm{e}-7$ \\
\hline 8 & $1.00 \ldots$ & $1.00004_{795} \ldots$ & $3.1 \mathrm{e} 12$ & & $1.95 \mathrm{e}-6$ \\
\hline 9 & $0.613 \ldots$ & $0.613_{79982 \ldots} \ldots$ & $9.0 \mathrm{e} 12$ & & $1.16 \mathrm{e}-6$ \\
\hline 10 & $0.486 \ldots$ & $0.49619_{468} \ldots$ & $7.3 \mathrm{e} 11$ & & $1.98 \mathrm{e}-6$ \\
\hline 11 & $0.994 \ldots$ & $1.00582_{237} \ldots$ & $7.3 \mathrm{e} 11$ & & $3.96 \mathrm{e}-6$ \\
\hline
\end{tabular}

Jukes in $[9,10]$ are also tabulated for comparison (the computation of $I_{10}$ and $I_{11}$ performed in [9] apparently contained a flaw, which is confirmed quickly even by evaluating the two truncated integrals verbatim, e.g. in Mathematica ${ }^{\circledR}$ ).

\section{Acknowledgements}

I would like to thank Steven Finch (Harvard University) for introducing me to the problem of computing the Tauberian constants $I_{k}$, as well as Dr. Jan van de Lune (Hallum, The Netherlands) and Professor Roger Heath-Brown FRS (Oxford University) for many instructive discussions and suggestions.

\section{References}

[1] Möbius, A.F., 1832, Über eine besondere Art von Umkehrung der Reihen. Journal für die Reine und Angewandte Mathematik, 9, 105-123.

[2] Riemann, B., 1859, Ueber die Anzahl der Primzahlen unter einer gegebenen Grösse. Monatsberichte der Preussischen Akademie der Wissenschaften, 1859, 671-680.

[3] Cohen, H., and Dress, F., 1988, Estimations numériques du reste de la fonction sommatoire relative aux entiers sans facteur carré. Publications Mathématiques d'Orsay, 88/02, 73-76.

[4] El Marraki, M., 1995, Fonction sommatoire de la fonction $\mu$ de Möbius: majorations asymptotiques effectives fortes. Journal de Théorie des Nombres de Bordeaux, 7, 407-433.

[5] Titchmarsh, E.C., 1951, The Theory of the Riemann Zeta Function (Oxford: Oxford University Press).

[6] Ng, N., 2004, The distribution of the summatory function of the Möbius function. Proceedings of the London Mathematical Society, 89, 361-389.

[7] Jukes, K.A., 1975, Tauberian constants connected with the prime number theorem. Mathematische Zeitschrift, 145, 187-193.

[8] Jukes, K.A., 1978, Equiconvergence of matrix transformations. Proceedings of the American Mathematical Society, 69, 261-270.

[9] Gretton, H.W., and Jukes, K.A., 1978, Tauberian constants connected with the prime number theorem. II. Computational aspects. Mathematische Zeitschrift, 164, 25-30.

[10] Gretton, H.W., and Jukes, K.A., 1979, Computations of Tauberian constants connected with equiconvergence and the quadratfrei numbers. Journal of Computational and Applied Mathematics, 5, 25-27.

[11] Lioen, W.M., and van de Lune, J., 1994, Systematic computations on Mertens' conjecture and Dirichlet's divisor problem by vectorized sieving. In: K. Apt, L. Schrijver, and N. Temme (Eds) From Universal Morphisms to Megabytes: A Baayen Space Odyssey (Amsterdam: CWI).

[12] Kotnik, T., and van de Lune, J., 2003, Further systematic computations on the summatory function of the Möbius function. CWI Report, MAS-R0313, 1-9.

[13] Kotnik, T., and van de Lune, J., 2004, On the order of the Mertens function. Experimental Mathematics, 13, 473-481.

[14] Good, I.J., and Churchhouse, R.F., 1968, The Riemann hypothesis and pseudorandom features of the Möbius sequence. Mathematics of Computation, 22, 857-861.

[15] Saffari, B., 1970, Sur la fausseté de la conjecture de Mertens. Avec une observation par Paul Lévy. Comptes Rendus de I'Académie des Sciences, 271A, 1097-1101. 